\title{
Comparison of Cable Method and Miniaci Method Using Picture Archiving and Communication System in Preoperative Planning for Open Wedge High Tibial Osteotomy
}

\author{
Seong-Dae Yoon, $\mathrm{MD}^{1}$, GuoFeng Zhang, $\mathrm{MD}^{2}$, Hee-June Kim, $\mathrm{MD}^{1}$, Byoung-Joo Lee, $\mathrm{MD}^{1}$, and \\ Hee-Soo Kyung, MD, $\mathrm{PhD}^{1}$ \\ ${ }^{1}$ Department of Orthopedic Surgery, Kyungpook National University Hospital, Daegu, Korea; ${ }^{2}$ Department of Orthopedic Surgery, Yantaishan Hospital, Yantai, China
}

\begin{abstract}
Purpose: The purpose was to compare the accuracy of Miniaci method using picture archiving and communication system (PACS) with a cable method in high tibial osteotomy (HTO).

Materials and Methods: This study analyzed 47 patients (52 knees) with varus deformity and medial osteoarthritis. From 2007 to 2013 , patients underwent HTO using either a cable method (20 knees) or Miniaci method based on a PACS image (32 knees). In the cable method, the 62.5\% point of the mediolateral tibial plateau width was located using an electrocautery cord under fluoroscopy (cable group). The Miniaci method used preoperative radiographs to shift the weight bearing axis (PACS group). Full-length lower limb radiographs obtained preoperatively and at the sixth postoperative week were used to compare the percentage of crossing point of the weight bearing line on the tibial plateau with respect to the medial border.

Results: The weight bearing line on the tibial plateau was corrected from a preoperative $11.0 \pm 7.0 \%$ to a postoperative $47.2 \pm 7.4 \%$ in the cable group and from $12.7 \pm 4.9 \%$ to $59.5 \pm 5.3 \%$ in the PACS group. The mechanical femorotibial angle was corrected from varus $8.9 \pm 3.7^{\circ}$ to valgus $0.3 \pm 4.0^{\circ}$ in the cable group and from varus $9.0 \pm 3.3^{\circ}$ to valgus $2.9 \pm 2.6^{\circ}$ in the PACS group.

Conclusions: In HTO, correction based on the Miniaci method using a PACS was more accurate than correction using the cable method.
\end{abstract}

Keywords: Knee, Osteotomy, Accuracy, Planning technique

\section{Introduction}

In patients with medial knee osteoarthritis with varus deformity, high tibial osteotomy (HTO) provides excellent results ${ }^{1-4)}$. Preoperative planning for appropriate correction gap and angle has a significant impact on the outcomes of $\mathrm{HTO}^{5-7)}$. One method used

Received September 13, 2016; Revised (1st) October 13, 2016;

(2nd) October 28, 2016; Accepted November 7, 2016

Correspondence to: Hee-Soo Kyung, MD, PhD

Department of Orthopedic Surgery, Kyungpook National University

Hospital, 130 Dongdeok-ro, Jung-gu, Daegu 41944, Korea

Tel: +82-53-200-5636, Fax: +82-53-422-6605

E-mail: hskyung@knu.ac.kr

This is an Open Access article distributed under the terms of the Creative Commons Attribution Non-Commercial License (http://creativecommons.org/licenses/by-nc/4.0/) which permits unrestricted non-commercial use, distribution, and reproduction in any medium, provided the original work is properly cited. for preoperative planning in HTO requires an intraoperative assessment of the lower limb mechanical axis with a radiopaque rod or an electrocautery cord (cable method). Another method involves an assessment of preoperative full-length weight bearing lower extremity radiographs with picture archiving and communication system (PACS) ${ }^{8)}$ or software, such as PreOPlan (Siemens, Munich, Germany/Synthes, Oberdorf, Switzerland) or mediCAD (Hectec GmbH, Altfraunhofen, Germany) ${ }^{9,10)}$. The cable method allows for real-time monitoring, but it can lead to unsatisfactory results due to the non-weight bearing status during surgery and increase radiation exposure of the surgeon and patient. In contrast, the Miniaci method using PACS calculates the correction angle and gap in weight bearing status, and thus more accurate values can be obtained with less radiation exposure without the need to detect the hip and ankle joint centers.

We hypothesized that correction of an angular deformity based 
on the weight bearing line would be more accurate and that the extent of preoperative deformity would affect postoperative correction. The purpose of this study was to compare the accuracy of the cable method and Miniaci method using a PACS in determining the correction angle in open wedge high tibial osteotomy (OWHTO) and to evaluate the correlation between the preoperative severity of varus deformity and outcomes.

\section{Materials and Methods}

\section{Study Design and Patient Selection}

This retrospective study analyzed 47 consecutive patients (52 knees) with varus deformity and medial knee osteoarthritis. From January 2007 to March 2013, patients underwent OWHTO using either the cable method (cable group: 20 patients [20 knees]) or the Miniaci method using PACS images (PACS group: 27 patients [32 knees]). The male-to-female ratio and mean age of patients were similar between groups (Table 1). Patients underwent medial OWHTO with the Aescula plate (B.Braun Korea, Seoul, Korea) in the cable group and the TomoFix plate (Synthes, Solothan, Swiss) in the PACS group. The inclusion criteria were as follows: 1 ) $<65$ years of age, 2 ) body mass index (BMI) of $<30$

Table 1. Comparative Results of Cable Method and Miniaci Method Using PACS

\begin{tabular}{lccc}
\hline \multicolumn{1}{c}{ Variable } & Cable method & $\begin{array}{c}\text { Miniaci method } \\
\text { using PACS }\end{array}$ & p-value \\
\hline No. of knees & 20 & 32 & \\
Age $(\mathrm{yr})$ & $55.2 \pm 7.7$ & $55 \pm 3.9$ & 0.912 \\
Sex $(\mathrm{M}: \mathrm{F})$ & $5: 15$ & $5: 27$ & 0.404 \\
Weight bearing line (\%) & & & \\
Preoperative & $11.0 \pm 7$ & $12.7 \pm 4.9$ & 0.511 \\
Postoperative & $47.2 \pm 7.4$ & $59.5 \pm 5.3$ & 0.018 \\
Weight bearing line & & & \\
Acceptable & 11 & 23 & 0.021 \\
Undercorrection & 8 & 3 & \\
Overcorrection & 1 & 6 & \\
mFTA $\left(^{\circ}\right)$ & & & \\
Preoperative & Varus $8.9 \pm 3.7$ & Varus $9.0 \pm 3.3$ & 0.213 \\
Postoperative & Valgus $0.3 \pm 4.0$ & Valgus $2.9 \pm 2.6$ & 0.017 \\
PTS angle $\left({ }^{\circ}\right)$ & & & \\
Preoperative & $9.5 \pm 2.3$ & $9.8 \pm 2.5$ & 0.675 \\
Preoperative & $10.4 \pm 2.2$ & $10.5 \pm 2.3$ & 0.869 \\
\hline
\end{tabular}

Values are presented as mean \pm standard deviation or number. PACS: picture archiving and communication system, mFTA: mechanical femorotibial angle, PTS: posterior tibial slope. $\mathrm{kg} / \mathrm{m}^{2}, 3$ ) pain in the medial side only, 4) high level of activities except for running and jumping, 5) varus angle of $<15^{\circ}$, and 6) knee flexion of $>90^{\circ}$. The exclusion criteria were as follows: 1 ) flexion contracture of $>25^{\circ}, 2$ ) bicompartmental disease, 3) elderly patients with difficulty in mobility, 4) BMI of $>30 \mathrm{~kg} / \mathrm{m}^{2}$, and 5) subtotal or total meniscectomy in the compartment near the planned osteotomy site. Patients who underwent a partial meniscectomy were not excluded.

\section{Preoperative Planning and Surgical Techniques}

In the preoperative planning stage, anteroposterior full-length lower limb weight bearing radiography was performed in both groups with a goal of achieving the target alignment passing through the Fujisawa point ${ }^{11)}$; that is, realigning the mechanical axis of the limb to be located at the $62.5 \%$ point from the medial border along the longest medial-to-lateral width of the tibial plateau. Both groups underwent diagnostic knee arthroscopy prior to osteotomy to verify conditions of the medial, lateral, and patellofemoral articular surfaces, which was followed by debridement. The superficial medial collateral ligament and pes anserinus were completely separated in both groups. In the cable group, a biplanar osteotomy was performed behind the tibial tuberosity. Then, the posteromedial tibial osteotomy site was opened using a chisel and a bone spreader, and the hip and ankle centers were connected using an electrocautery cord under fluoroscopic guidance. The osteotomy site was spread until the electrocautery cord could be placed at the target point on the medial-to-lateral tibial plateau of the knee joint while applying axial load at the foot sole. Then, the metal plate was fixed (Fig. 1) ${ }^{1)}$. The PACS group underwent anteroposterior full-length lower limb radiography with valgus stress applied to the bilateral knee joints under weight bearing condition in order to correct widening of the lateral joint space induced by ligament laxity. Using the preoperative radiograph, the lower limb weight bearing line was drawn (line 1, S). After calculating the $62.5 \%$ point from the medial border along the longest medial-to-lateral width of the tibial plateau, an extension line connecting the hip center and the target point (line 2, S') was drawn. Then, a line connecting the lateral tibial osteotomy site (D) and the center of the ankle joint (line 3, DS) and a line connecting the osteotomy site and S' (line 4, DS') were drawn (Fig. 2A). The angle formed by lines 3 and 4 was determined to be the predicted correction wedge angle $(\alpha)$. A predicted osteotomy line (O) was drawn from the proximal extremity of the fibular head to the predicted medial osteotomy site (approximately $4 \mathrm{~cm}$ inferior to the medial border of the tibial plateau), and a predicted opening line $\left(\mathrm{O}^{\prime}\right)$ was drawn from Line $\mathrm{O}$ at the determined correction angle (wedge angle, $\alpha$ ). Using the triangle formed, we 

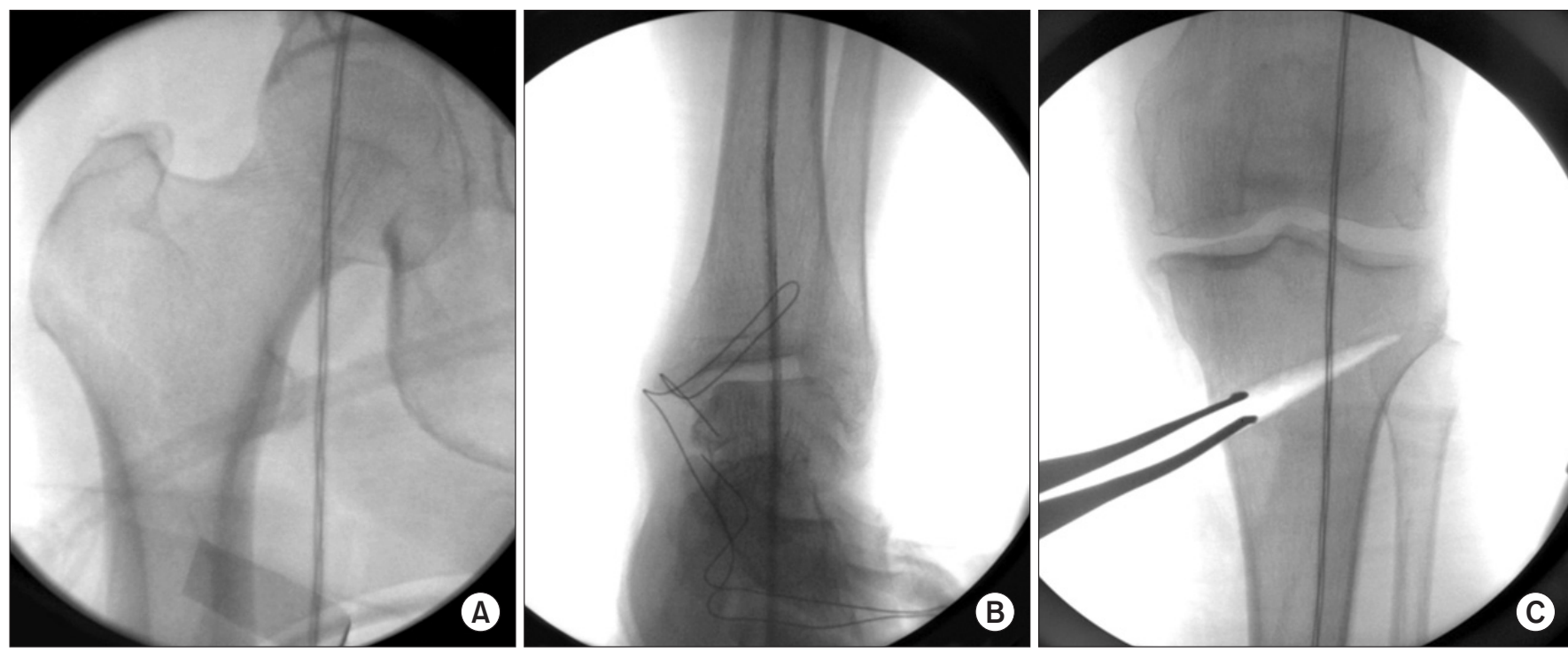

Fig. 1. Cable method. (A) The center of the hip was identified with fluoroscopy. (B) The center of the ankle was identified with fluoroscopy. (C) The hip and ankle centers were connected using an electrocautery cord under fluoroscopic guidance. The osteotomy site was spread until the electrocautery cord was placed at the target point on the medial-to-lateral tibial plateau of the knee joint. Then, the metal plate was fixed.
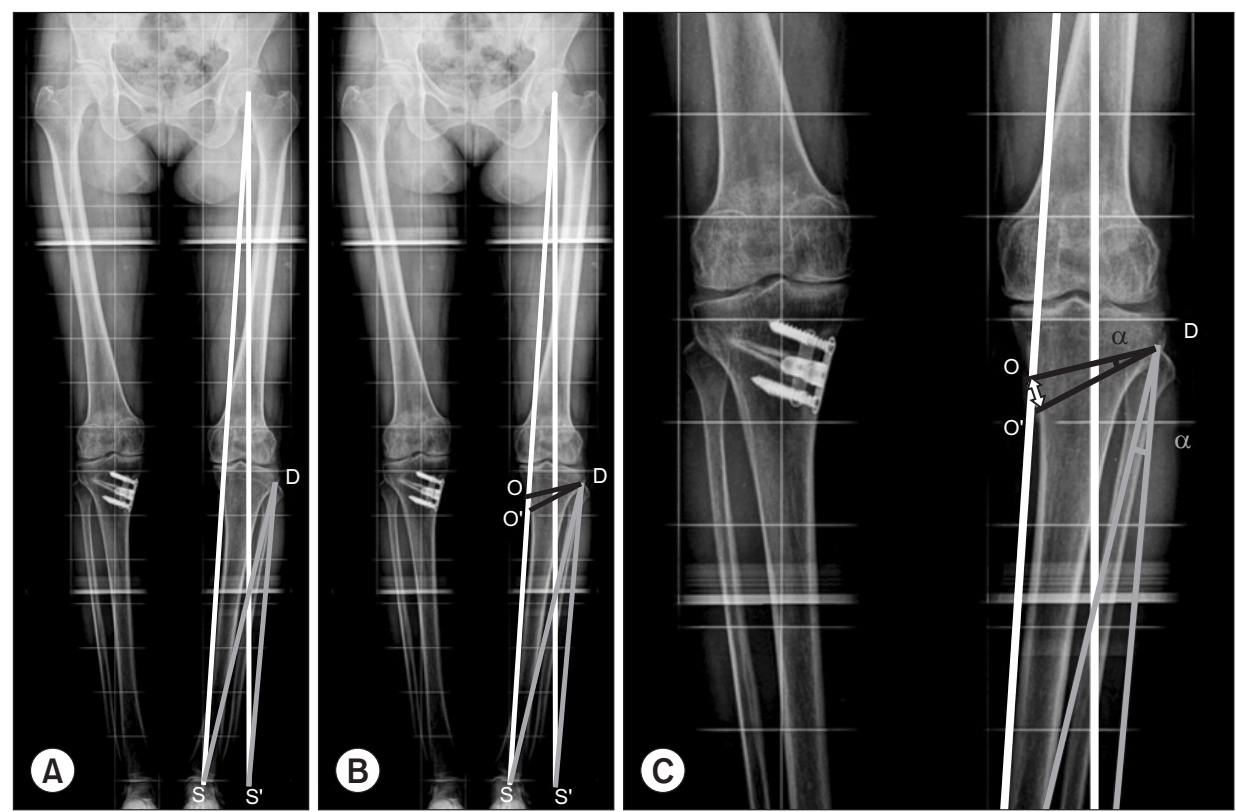

Fig. 2. Miniaci method using a picture archiving and communication system. (A) On the preoperative anteroposterior full-length lower limb radiograph, the lower limb weight bearing line (line 1, S) was drawn. After calculating the $62.5 \%$ point from the medial border along the longest medial-tolateral width of the tibial plateau, an extension line connecting the hip center and the calculated point (line 2, S') was drawn. Then, a line connecting the lateral tibial osteotomy site (D) and the center of the ankle joint (line 3, DS) was drawn. Another line connecting the osteotomy site and line 2 (line 4 , DS') was drawn. The angle formed by lines 3 and 4 was determined to be the predicted correction angle. (B, C) A predicted osteotomy line (O) was drawn from the proximal extremity of the fibular head to the predicted medial osteotomy site (approximately $4 \mathrm{~cm}$ inferior to the medial border of the tibial plateau). A predicted opening line $\left(\mathrm{O}^{\prime}\right)$ was drawn from Line $\mathrm{O}$ at the determined correction angle (wedge angle, $\alpha$ ). The predicted correction gap (wedge gap, $\mathrm{mm}$ ) at the cortical bone of the posteromedial tibia was measured.

measured the predicted correction gap (wedge gap, $\mathrm{mm}$ ) at the cortical bone of the posteromedial tibia (Fig. $2 \mathrm{~B}$ and C) ${ }^{12}$. After performing an osteotomy using the same surgical technique as in the cable group, we spread the osteotomy site matching the predicted wedge angle and gap and fixed the metal plate. Allogeneic bone and autologous bone marrow harvested from the anterior 
superior iliac spine on the same side were mixed and grafted onto the bone defect. The grafted site was covered with the superficial medial collateral ligament, and the pes anserinus was resutured to the periosteal membrane. An active exercise program for joint rehabilitation began in the second postoperative week. The Aescula plate group (cable group) and TomoFix plate group (PACS group) were allowed to commence body weight bearing in the sixth and second postoperative weeks, respectively.

\section{Evaluation and Measurement}

The full-length lower limb radiographs obtained preoperatively and at the sixth postoperative week were used to compare the percentage of crossing point of the weight bearing line on the tibial plateau with respect to the medial border. The acceptable postoperative range was set at $62 \% \pm 5 \%$ (range, $57 \%$ to $67 \%$ ), and percentages lower or higher than this range were defined as overcorrection or undercorrection, respectively ${ }^{13)}$. Pre- and postoperative mechanical femorotibial angles (mFTA) and posterior tibial slope angles were also compared ${ }^{14)}$. In the PACS group, the preoperatively predicted and postoperatively measured wedge angles and gaps were compared. The wedge gaps were measured as the distance between line $\mathrm{O}$ and line $\mathrm{O}^{\prime}$ at the medial cortex, and the wedge angles as the angle formed by the two lines.

This study was approved by the Institutional Review Board of our institution (no. 2014-09-037-001).

\section{Statistical Analysis}

A two-sample $t$-test was used to compare the mean values of age, weight bearing line, and mFTA between groups. Chi-square analysis was used to compare the categorical variables between the two groups. Logistic regression analysis was used to evaluate the relationship between the preoperative severity of varus deformity and the outcome of correction based on the location of the weight bearing line on the tibial plateau. Radiographic measurements were performed by two observers. The correlation coefficient between the two observers was 0.79 when calculated by Spearman's rho test. The mean values of two measurements were used for analysis.

Statistical significance was assumed at $\mathrm{p}<0.05$. All statistical analyses were performed with SPSS ver. 19.0 (IBM Co., Armonk, NY, USA).

\section{Results}

The weight bearing line on the tibial plateau was corrected from a preoperative mean of $11.0 \% \pm 7.0 \%$ to a postoperative mean of $47.2 \% \pm 7.4 \%$ in the cable group and from a mean of $12.7 \% \pm 4.9 \%$ to a mean of $59.5 \% \pm 5.3 \%$ in the PACS group ( $\mathrm{p}=0.018$ ). The ratio of undercorrection, acceptable range, and overcorrection was 8:11:1 in the cable group and 3:23:6 in the PACS group. The Miniaci method using PACS images resulted in a significantly higher incidence of correction in the acceptable range than did the cable method $(\mathrm{p}=0.021)$ (Table 1$)$.

The mFTA was corrected from a preoperative mean of varus $8.9^{\circ} \pm 3.7^{\circ}$ to a postoperative mean of valgus $0.3^{\circ} \pm 4.0^{\circ}$ in the cable group and from a mean of varus $9.0^{\circ} \pm 3.3^{\circ}$ to a mean of valgus $2.9^{\circ} \pm 2.6^{\circ}$ in the PACS group. The postoperative difference between the two groups was significant $(\mathrm{p}=0.017)$. The posterior tibial slope angle was increased from $9.5^{\circ} \pm 2.3^{\circ}$ to $10.4^{\circ} \pm 2.2^{\circ}$ $(\mathrm{p}<0.001)$ in the cable group and from $9.8^{\circ} \pm 2.5^{\circ}$ to $10.5^{\circ} \pm 2.3^{\circ}$ $(\mathrm{p}<0.001)$ in the PACS group. The preoperative and postoperative differences were not significant $(\mathrm{p}=0.675$ and $\mathrm{p}=0.869$, respectively) (Table 1).

In the PACS group, 18 cases with undercorrection fell short of the target angle by a mean of varus $1.8^{\circ} \pm 1.9^{\circ}$ while 14 cases with overcorrection exceeded the target angle by a mean of valgus $2.6^{\circ} \pm 2.0^{\circ}$. The overall differences between the preoperatively planned and postoperatively achieved wedge gaps and angles by absolute values were a mean of $3.6 \pm 5.2 \mathrm{~mm}$ and $2.1^{\circ} \pm 1.9^{\circ}$, respectively (Table 2 ). There was no significant correlation between the preoperative severity of varus deformity and postoperative outcomes based on the location of the weight bearing line on the tibial plateau $(\mathrm{p}=0.477)$.

\section{Discussion}

In this study, correction of angular deformity by the Miniaci method using PACS images was more accurate than correction by the cable method in OWHTO.

For osteotomy aimed at mechanical axis realignment, preoperative correction planning is of pivotal importance for successful surgical outcome ${ }^{15,16)}$. Many authors have presented various pre-

Table 2. Difference between Preoperatively Predicted and Postoperatively Measured Wedge Angles and Gaps in the PACS Group

\begin{tabular}{lcc}
\hline \multicolumn{1}{c}{ Variable } & Wedge angle $\left(^{\circ}\right)$ & Wedge gap $(\mathrm{mm})$ \\
\hline Undercorrection $(<62.5 \%, \mathrm{n}=18)$ & Varus $1.8 \pm 1.9$ & Varus 3.0 \pm 2.3 \\
Overcorrection $(>62.5 \%, \mathrm{n}=14)$ & Valgus 2.6 \pm 2.0 & Valgus $4.4 \pm 7.5$ \\
Total $^{\mathrm{a}}(\mathrm{n}=32)$ & $2.1 \pm 1.9$ & $3.6 \pm 5.2$ \\
\hline
\end{tabular}

Values are presented as mean \pm standard deviation.

PACS: picture archiving and communication system.

a) The overall differences of absolute values. 
operative planning modalities ${ }^{12,17-19)}$. Still, the radiopaque line is frequently employed for intraoperative guidance. This method is likely to lead to an unacceptable range of correction after surgery and increase radiation exposure of both the surgeon and patient due to the increased duration of radiography for identifying the hip and ankle joint centers and for verifying the degree of correction. A navigation-based technique has recently been reported, and its accuracy and superiority have been confirmed in clinical trials $^{20-23)}$. However, this method involves the use of navigation devices and is applied intraoperatively under non-weight bearing condition, which can yield deviating correction angles after surgery under weight bearing condition. Apart from this accuracy issue, controversy surrounds the cost-effectiveness of the method, which was kindled by research suggesting it has no clinical advantage over conventional methods ${ }^{24)}$.

Preoperative planning can be implemented based on the anatomic axis ${ }^{1)}$, mechanical axis ${ }^{6,12)}$, or the weight bearing line ${ }^{8,10)}$. The weight bearing line can reflect instability around the knee joint and deviate from the mechanical axis. As demonstrated by the results of this study, significant differences were observed between the cable-based method involving intraoperative fluoroscopic guidance using a radiopaque line and the PACS imagebased preoperative planning. The cable group tended to obtain an unacceptable correction because the mechanical axis was measured in a non-weight bearing supine position, whereas the PACS image-based method used the weight bearing line assessed in upright position as a reference. Differences in the mechanical axis between radiographs obtained in preoperative upright (weight bearing) position and those in supine position have been reported to be $\geq 3^{025)}$, presumably due to differences in the knee joint position or lateral laxity under weight bearing condition. To correct such deviations, a valgus stress can be applied during preoperative anteroposterior full-length lower limb radiography ${ }^{13)}$. In this study, the cable group had a high ratio of undercorrection, whereas Lee and Moon ${ }^{26)}$ reported the cable method may result in rather overcorrected alignment because the MCL is measured in a state of relaxation. In the current study, axial pressure was applied to the foot sole for simulating the weight bearing state during operation performed using the cable method. This caused decreased lateral laxity, resulting in undercorrection than preoperatively planned. However, when weight bearing was applied postoperatively, lateral laxity recurred due to muscular action. Moreover, limb rotation during intraoperative fluoroscopy may have resulted in the variation on postoperative weight bearing radiographs and the wide deviation of the correction angle from the target angle in the cable group.
Only 23 cases (71.8\%) in the PACS group had the weight bearing line within the acceptable range because the radiographs were not obtained from the same fixed position and differences in bone quality may have contributed to undercorrection during intraoperative gap spreading. Also, the thickness of the saw blade could have affected postoperative undercorrection.

This study had some limitations. First, the number of cases was small. Second, the study did not investigate clinical outcomes. Therefore, a clinical study should be conducted in a large series. Radiographs were not obtained from the same fixed position, which could have undermined the reliability of radiographic measurements considering this can influence postoperative radiographic values. Finally, different plates were used in the two groups. However, taking consideration into the difference in implant stability, weight bearing was allowed at 6 weeks after surgery in the cable group and at 2 weeks after surgery in the PACS group; therefore, the type of plate might not have much influence on correction loss at the time of the sixth postoperative week radiographic evaluation.

\section{Conclusions}

In OWHTO, correction of angular deformity based on the Miniaci method using a PACS was more accurate than correction using the cable method. Also this method can be expected to involve less intraoperative radiation exposure. There was no significant correlation between the preoperative severity of varus deformity and outcomes based on the location of the weight bearing line on the tibial plateau. Therefore, the Miniaci method using PACS can be useful in preoperative planning for OWHTO.

\section{Conflict of Interest}

No potential conflict of interest relevant to this article was reported.

\section{References}

1. Amendola A, Bonasia DE. Results of high tibial osteotomy: review of the literature. Int Orthop. 2010;34:155-60.

2. Ozalay M, Ozkoc G, Circi E, Akpinar S, Hersekli MA, Uysal $\mathrm{M}$, Cesur N. The correlation of correction magnitude and tibial slope changes following open wedge high tibial osteotomy. Knee Surg Sports Traumatol Arthrosc. 2008;16:948-51.

3. Song EK, Seon JK, Park SJ, Jeong MS. The complications of high tibial osteotomy: closing- versus opening-wedge meth- 
ods. J Bone Joint Surg Br. 2010;92:1245-52.

4. Sprenger TR, Doerzbacher JF. Tibial osteotomy for the treatment of varus gonarthrosis. Survival and failure analysis to twenty-two years. J Bone Joint Surg Am. 2003;85:469-74.

5. Engel GM, Lippert FG 3rd. Valgus tibial osteotomy: avoiding the pitfalls. Clin Orthop Relat Res. 1981;(160):137-43.

6. Kettelkamp DB, Wenger DR, Chao EY, Thompson C. Results of proximal tibial osteotomy. The effects of tibiofemoral angle, stance-phase flexion-extension, and medial-plateau force. J Bone Joint Surg Am. 1976;58:952-60.

7. Tjornstrand B, Selvik G, Egund N, Lindstrand A. Roentgen stereophotogrammetry in high tibial osteotomy for gonarthrosis. Arch Orthop Trauma Surg. 1981;99:73-81.

8. Marx RG, Grimm P, Lillemoe KA, Robertson CM, Ayeni OR, Lyman S, Bogner EA, Pavlov H. Reliability of lower extremity alignment measurement using radiographs and PACS. Knee Surg Sports Traumatol Arthrosc. 2011;19:1693-8.

9. Heijens E, Kornherr P, Meister C. The role of navigation in high tibial osteotomy: a study of 50 patients. Orthopedics. 2009;32(10 Suppl):40-3.

10. Schroter S, Ihle C, Mueller J, Lobenhoffer P, Stockle U, van Heerwaarden R. Digital planning of high tibial osteotomy: interrater reliability by using two different software. Knee Surg Sports Traumatol Arthrosc. 2013;21:189-96.

11. Fujisawa Y, Masuhara K, Shiomi S. The effect of high tibial osteotomy on osteoarthritis of the knee: an arthroscopic study of 54 knee joints. Orthop Clin North Am. 1979;10:585608.

12. Miniaci A, Ballmer FT, Ballmer PM, Jakob RP. Proximal tibial osteotomy: a new fixation device. Clin Orthop Relat Res. 1989;(246):250-9.

13. Marti CB, Gautier E, Wachtl SW, Jakob RP. Accuracy of frontal and sagittal plane correction in open-wedge high tibial osteotomy. Arthroscopy. 2004;20:366-72.

14. Hernigou P, Medevielle D, Debeyre J, Goutallier D. Proximal tibial osteotomy for osteoarthritis with varus deformity: a ten to thirteen-year follow-up study. J Bone Joint Surg Am. 1987;69:332-54.

15. El-Azab HM, Morgenstern M, Ahrens P, Schuster T, Imhoff AB, Lorenz SG. Limb alignment after open-wedge high tibial osteotomy and its effect on the clinical outcome. Orthopedics. 2011;34:e622-8.
16. Marti RK, Verhagen RA, Kerkhoffs GM, Moojen TM. Proximal tibial varus osteotomy. Indications, technique, and five to twenty-one-year results. J Bone Joint Surg Am. 2001;83: 164-70.

17. Coventry MB. Upper tibial osteotomy for osteoarthritis. J Bone Joint Surg Am. 1985;67:1136-40.

18. Dugdale TW, Noyes FR, Styer D. Preoperative planning for high tibial osteotomy: the effect of lateral tibiofemoral separation and tibiofemoral length. Clin Orthop Relat Res. 1992; (274):248-64.

19. Pape D, Seil R, Adam F, Rupp S, Kohn D, Lobenhoffer P. Imaging and preoperative planning of osteotomy of tibial head osteotomy. Orthopade. 2004;33:122-34.

20. Blakeney WG, Khan RJ, Wall SJ. Computer-assisted techniques versus conventional guides for component alignment in total knee arthroplasty: a randomized controlled trial. J Bone Joint Surg Am. 2011;93:1377-84.

21. Goleski P, Warkentine B, Lo D, Gyuricza C, Kendoff D, Pearle AD. Reliability of navigated lower limb alignment in high tibial osteotomies. Am J Sports Med. 2008;36:2179-86.

22. Hankemeier S, Hufner T, Wang G, Kendoff D, Zeichen J, Zheng G, Krettek C. Navigated open-wedge high tibial osteotomy: advantages and disadvantages compared to the conventional technique in a cadaver study. Knee Surg Sports Traumatol Arthrosc. 2006;14:917-21.

23. Hankemeier S, Hufner T, Wang G, Kendoff D, Zheng G, Richter M, Gosling T, Nolte L, Krettek C. Navigated intraoperative analysis of lower limb alignment. Arch Orthop Trauma Surg. 2005;125:531-5.

24. Kyung BS, Kim JG, Jang KM, Chang M, Moon YW, Ahn JH, Wang JH. Are navigation systems accurate enough to predict the correction angle during high tibial osteotomy? Comparison of navigation systems with 3-dimensional computed tomography and standing radiographs. Am J Sports Med. 2013;41:2368-74.

25. Sabharwal S, Zhao C. Assessment of lower limb alignment: supine fluoroscopy compared with a standing full-length radiograph. J Bone Joint Surg Am. 2008;90:43-51.

26. Lee YS, Moon GH. Comparative analysis of osteotomy accuracy between the conventional and devised technique using a protective cutting system in medial open-wedge high tibial osteotomy. J Orthop Sci. 2015;20:129-36. 